\title{
Reestructuración preventiva frente a insolvencia. Aproximación didáctica a la novísima normativa de la Unión Europea (2019) ilustrada por la xilografía de Katsushika Hokusai (1760-1849)
}

\author{
Huerta Viesca, $M^{\text {a }}$. Isabel
}

Universidad de Oviedo, España

\begin{abstract}
Resumen
Para desarrollar una educación que propicie una sociedad más crítica, justa e igualitaria es necesario contar con material didáctico que facilite la incorporación de perspectivas transversales en todas las materias. Ello fomentará el aprendizaje reflexivo y duradero. En concreto, en la docencia del Derecho preconcursal, tanto en el ámbito universitario como previo -en la Enseñanza Secundaria, el Bachillerato y la Formación Profesional-puede ser valioso el recurso didáctico a la pintura y la xilografía, que permitirán ilustrar los principios y las connotaciones sociales, económicas y jurídicas de esta parcela jurídica y que favorecerá un enfoque vivamente crítico sobre la materia societaria y de la crisis económica de los empresarios y de los particulares. Este trabajo atiende a esta innovadora propuesta docente.
\end{abstract}

Palabras clave: insolvencia; reestructuración preventiva; Derecho concursal; crisis económica; Derecho de la empresa; Derecho mercantil; pintura; docencia; sociedad justa e igualitaria; xilografía; Katsushika Hokusai

\section{Introducción}

La situación de insolvencia inminente para un consumidor o para un empresario significa casi siempre, para quien la sufre, verse engullido por una ola gigante de la que probablemente saldrá malparado, puesto que las soluciones que ha venido ofreciendo hasta ahora el Derecho para estos casos, como ha demostrado la grave crisis económica que acabamos de padecer, no son eficaces para resolver los problemas que se generan en el entorno familiar o empresarial y no impiden la expansión de estas situaciones, con un "efecto onda", a la economía y a la sociedad en su conjunto. 


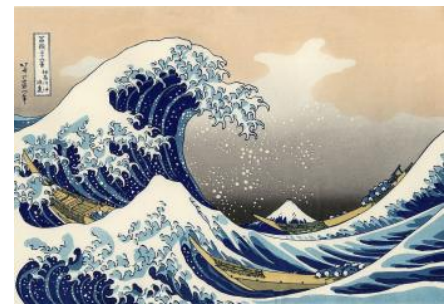

Hokusai, La gran ola de Kanagawa (1830) ${ }^{1}$

Los procedimientos concursales existentes tradicionales, en España y en el resto de la Unión Europea, no dan una solución a las situaciones de crisis e insolvencia, ni a los consumidores, ni a las familias, ni a los empresarios. Este trabajo propone una reflexión introductoria muy básica, especialmente dirigida a discentes anteriores a los estudios de Grado, sobre la materia de la reestructuración preventiva como mecanismo para evitar la insolvencia y el concurso. La insolvencia y la evitación de la insolvencia son materias de gran importancia teórica $\mathrm{y}$, sobre todo, práctica. Nos afectan, directa o indirectamente a todos los ciudadanos e incluso pueden determinar la evitación de crisis económicas como las que acabamos de padecer. Como entiende que los mecanismos de reestructuración preventiva son capitales y que trascienden, con mucho, la perspectiva de cada uno de los Estados miembros, la Unión Europea ha dictado recientemente una normativa comunitaria, con contenido de alcance general y obligatorio para todos los Estados miembros, que tratará de aumentar de forma significativa la eficacia de los procedimientos de condonación, insolvencia y reestructuración. Esta propuesta, pues, puede ser especialmente valiosa en la docencia relacionada con los empresarios, con la economía y con las empresas en cualquier Grado, Formación Profesional o Bachillerato, en cuanto dará a conocer, aunque sea ahora sucintamente y en líneas muy generales y únicamente introductorias, los elementos esenciales y las características de la nueva regulación europea para los deudores en dificultades financieras cuando la insolvencia sea inminente, con el fin de evitar la insolvencia y también de garantizar la viabilidad del deudor.

Hasta aquí podría decirse que la perspectiva será introductoria a una cuestión eminentemente jurídica y empresarial. Pero la propuesta, en este momento, tiene un objetivo complementario también relevante: la ilustración, mediante obras artísticas de los principales problemas que origina la nueva normativa "pre" y "anti" crisis y "sobre marcos de reestructuración preventiva". Se trata de introducir un elemento didáctico anecdótico pero muy útil, puesto que podrá conducir a la fijación y comprensión de la situación actual en las situaciones de crisis y de las nuevas propuestas comunitarias desde la atención a las

\footnotetext{
${ }^{1}$ La conocida obra de Katsushika Hokusai, La gran ola de Kanagawa, que ilustra una tempestad en alta mar justo cuando va a impactar contra una barca, es expresiva de la situación de dificultad a la que se enfrentan los empresarios o particulares ante las situaciones de crisis económica o insolvencia inminente y del efecto expansivo de estas crisis en todo su entorno personal, económico y social.
} 
obras de la serie Treinta y Seis Vistas del Monte Fuji de Hokusai (1760-1849), propiciando a través de estos ejemplos visuales un aprendizaje duradero fundamentalmente en los alumnos de preuniversitario.

\section{Motivos que conducen a la Unión Europea a la adopción de la nueva Directiva sobre insolvencia}

El día 28 de marzo de 2019 se aprobó en Estrasburgo la Directiva del Parlamento Europeo y del Consejo sobre marcos de reestructuración preventiva, segunda oportunidad y medidas para aumentar la eficacia de los procedimientos de condonación, insolvencia y reestructuración, y por la que se modifica la Directiva 2012/30/UE (COM(2016)0723 - C8$0475 / 2016$ - 2016/0359(COD) $)^{2}$. ¿Qué significa esta normativa? En primer lugar hay que señalar qué es una Directiva comunitaria. Se trata de una norma de la Unión Europea muy original. Obliga a todos y cada uno de los Estados miembros de la Unión a adaptar su legislación interna, por los medios que cada Estado considere apropiados, al contenido de la propia Directiva y en un plazo fijado en ella misma. Si los Estados miembros incumplieran los plazos dados y no adaptaran su normativa interna a la Directiva, si ésta tiene un contenido claro, preciso e incondicional, los particulares podrían incluso solicitar en el curso de un juicio su aplicación directa.

Pues bien: la Unión Europea legisla, mediante esta Directiva de 2019, en la materia de los procedimientos de insolvencia y reestructuración previa al concurso. Frente a la divergencia de las legislaciónes de los Estados miembros, la Unión Europea se decide a regular de forma coordinada una materia tan sensible a la historia y a la cultura jurídica de cada uno de los Estados miembros tendiendo hacia su homogenización. Realmente la Directiva de 2019 marca un momento histórico, pues la Unión Europea contribuye por primera vez en este campo al logro de un mejor funcionamiento del mercado interior evitando a la vez los obstáculos al ejercicio de las libertades fundamentales que resultan de las regulaciones, tan dispares, de los Estados miembros. Concretamente, la Unión Europea se preocupa por la incertidumbre que genera la dispersión normativa sobre la materia de insolvencia y pre-insolvencia en el seno comunitario. Considera que esas divergencias normativas entre los entre los Estados miembros se traducen en costes adicionales para los inversores, que tendrán que evaluar, por ejemplo, el posible riesgo de que sus deudores vayan a sufrir dificultades financieras en uno o más Estados miembros. Y es aquí, en la

\footnotetext{
${ }^{2}$ Resolución legislativa del Parlamento Europeo, de 28 de marzo de 2019, sobre la Propuesta de Directiva del Parlamento Europeo y del Consejo sobre marcos de reestructuración preventiva, segunda oportunidad y medidas para aumentar la eficacia de los procedimientos de condonación, insolvencia y reestructuración, y por la que se modifica la Directiva 2012/30/UE (COM(2016)0723 - C8-0475/2016 - 2016/0359(COD)).
} 
cuestión de la incertidumbre, donde la Unión Europea justifica la necesidad de la Directiva, en cuanto observa que esa incertidumbre sí tiene un efecto disuasorio que obstaculiza la libertad de establecimiento de las empresas y el fomento del espíritu empresarial, a la vez que perjudica el funcionamiento correcto del mercado interior. Esa incertidumbre tiene consecuencias muy perniciosas, sobre todo, para las microempresas y pequeñas y medianas empresas, que no disponen generalmente de los recursos necesarios para evaluar los riesgos en que pueden incurrir cuando llevan a cabo actividades transfronterizas en el mercado interior. El fundamento para esa nueva regulación es el llamado "efecto dominó" de las crisis empresariales en el mercado único, en el que "la insolvencia de un deudor puede conllevar la insolvencia de otros varios eslabones del mercado" ${ }^{3}$ en una cadena sin final. En definitiva, todos estamos en el mismo barco: la crisis de unos, afectará tarde o temprano a todos los ciudadanos. Es necesario que el Derecho ofrezca soluciones prácticas eficaces cuanto antes se aprecien signos de alarma.

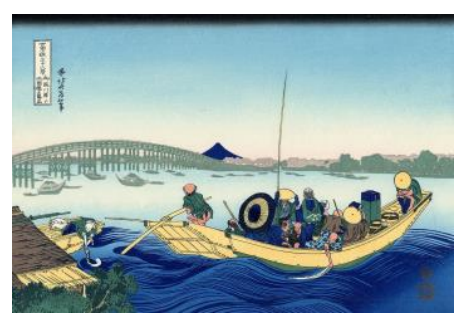

Hokusai, Puesta de sol a través del puente Ryogoku desde la orilla del río Sumida, en Onmagayashi (1830) ${ }^{4}$

Además la Unión Europea observa el rotundo fracaso de las soluciones jurídicas que se han venido dando por los sistemas jurídicos nacionales ante situaciones de insolvencia. Los procedimientos de reestructuración, insolvencia y exoneración de deudas tienen una excesiva duración y tienen costes demasiado elevados. Pasar por uno de los procedimientos actuales de reestructuración de deuda y de concurso (anterior quiebra) es realmente un desafío para quien sigue esa senda.

\footnotetext{
3 Así, el Considerando (11) de la Directiva establece que "los obstáculos al ejercicio de las libertades fundamentales no se limitan a las situaciones de naturaleza estrictamente transfronteriza. Un mercado interior cada vez más interconectado, en el que las mercancías, los servicios, los capitales y los trabajadores circulan libremente, y con una dimensión digital cada vez mayor supone que muy pocas empresas son estrictamente nacionales si se tienen en cuenta todos los elementos pertinentes, como la clientela, la cadena de suministro, el ámbito de actividad, los inversores y el capital. Incluso las insolvencias estrictamente nacionales pueden incidir en el funcionamiento del mercado interior a través del denominado «efecto dominó» de las insolvencias, mediante el cual la insolvencia de un deudor puede dar lugar a la insolvencia de otros eslabones de la cadena de suministro", llegando a todas las capas sociales sin excepción.

${ }^{4}$ Hokusai, K., Puesta de sol a través del puente Ryogoku desde la orilla del río Sumida, en Onmayagashi (1830). File:Sunset across the Ryogoku bridge from the bank of the Sumida river at Onmagayashi.jpg. (2014, July 24). Wikimedia Commons, the free $\begin{array}{llllll}\text { media } & \text { repository. } & \text { Retrieved } & \text { May } & 2019 & \text { from }\end{array}$ https://commons.wikimedia.org/w/index.php?title=File:Sunset_across_the_Ryogoku_bridge_from_the_bank_of_the_Sumida_river at_Onmagayashi.jpg\&oldid=129772361.
} 


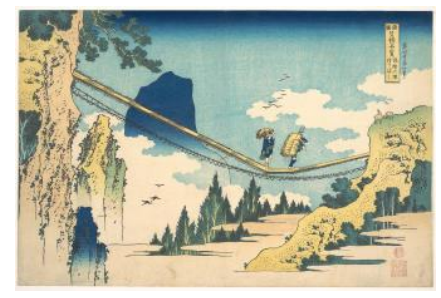

Hokusai, Puente colgante $(1830)^{5}$.

La imagen de Hokusai de unas personas cruzando el puente colgante es muy expresiva de la realidad actual ante estos procedimientos todavía vigentes. En total contraste, la Directiva comunitaria de 2019 diseña un marco preventivo para la reestructuración finanaciera cuando la insolvencia sea inminente, para garantizar la viabilidad del deudor y establece una serie de medidas que aumentarán en todos los Etados miembros la eficiencia de los procedimientos de reestructuración, insolvencia y exoneración de deudas.

\section{Medidas propuestas en la Directiva}

La Directiva de 2019 procura que no se vean afectados ni los derechos fundamentales de los trabajadores ni sus libertades. Entiende que es esencial un mayor grado de armonización de materia de reestructuración e insolvencia para conseguir una mayor resiliencia de la economía europea y especialmente para la creación y el mantenimiento de los puestos de trabajo. En lo que respecta a los trabajadores, la Directiva trata de reforzar su apoyo de modo que los Estados miembros velen por que se permita a los representantes de los trabajadores acceder a información pertinente y actualizada sobre la disponibilidad de herramientas de alerta temprana, gocen de información sobre la propuesta de plan de reestructuración a fin de que puedan efectuar una evaluación en profundidad de las distintas posibilidades y se preste asistencia a los representantes de los trabajadores a la hora de evaluar la situación económica del deudor. Los Estados miembros podrán excluir los créditos de los trabajadores del ámbito de aplicación de los marcos de reestructuración preventiva. Con el punto de mira en los trabajadores, se diseñan medidas preventivas.

\footnotetext{
5 Hokusai, K., Puente colgante (1830). The Suspension Bridge on the Border of Hida and Etchü Provinces (Hietsu no sakai tsuribashi), from the series Remarkable Views of Bridges in Various Provinces (Shokoku meikyō kiran). The Met. https://www.metmuseum.org/art/collection/search/53192
} 


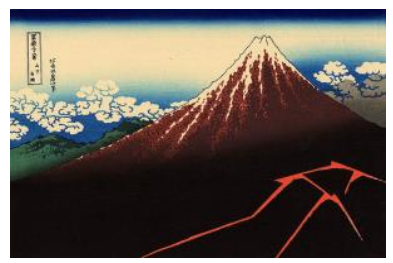

Hokusai, Tormenta debajo de la cumbre $(1830)^{6}$

En lugar de que el camino sea el seguido hasta ahora, en que la insolvencia lleva a la liquidación de la empresa, se abre una nueva vía: se tiende a favorecer planteamientos que tengan por objeto la recuperación de la empresa o de sus unidades económicamente viables. Tan pronto como se observen signos de alarma o de alerta deberán ponerse en marcha los mecanismos preventivos previstos para evitar la insolvencia y el concurso.

La reestructuración permitirá a los deudores en dificultades financieras continuar con su actividad empresarial, en su totalidad o en parte, modificando la composición, las condiciones o la estructura del activo y del pasivo o de cualquier otra parte de su estructura de capital, por ejemplo, en su caso mediante la venta de activos o de parte de la empresa.

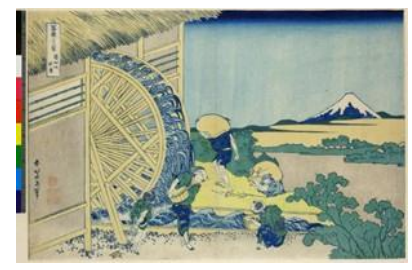

Hokusai, Rueda Hidráulica $(1830)^{7}$

La Directiva ofrece pues un puente para que las medidas de reestructuración permita superar la etapa de peligro de la insolvencia y la crisis económica.

\footnotetext{
${ }^{6}$ Hokusai, Tormenta debajo de la cumbre (1830), File:Lightnings below the summit.jpg. Wikimedia Commons, the free media $\begin{array}{llllll}\text { repository. } & \text { Retrieved } & 20: 24, & \text { May } & 24, & 2019\end{array}$ https://commons. wikimedia.org/w/index.php?title=File:Lightnings_below_the_summit.jpg\&oldid=72583332.

${ }^{7}$ La xiligrafía de Katsushika Hokusai Rueda Hidráulica o Molino de agua japonés en Onden (hacia 1830) en que los operarios siguen trabajando puede aqyudarnos a recordar la continuidad de la empresa y del mantenimiento de los puestos de trabajo durante la sustanciación de los nuevos procedimientos preventivos y ante la insolvencia inminente que instaura la Directiva de insolvencia de 2019. File:Watermill at Onden.jpg. (2015, May 6). Wikimedia Commons, the free media repository. Retrieved 19:40, May 24, 2019 from https://commons.wikimedia.org/w/index.php?title=File:Watermill_at_Onden.jpg\&oldid=159996240.
} 


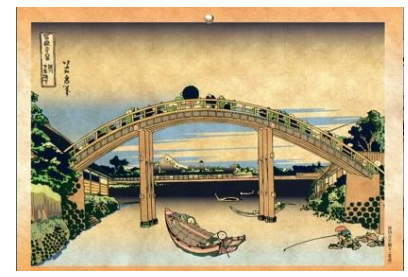

Hokusai, Bajo el puente Mannen en Fukagawa $(1832)^{8}$

En conclusión, los marcos de reestructuración preventiva permitirán, ante todo, la reestructuración efectiva de los deudores en un momento temprano y evitar la insolvencia, limitando la liquidación innecesaria de empresas viables. Con las nuevas medidas se ayudará a evitar la pérdida de puestos de trabajo y de conocimientos y competencias y se maximizará el valor total para los acreedores en comparación con lo que habrían recibido, por ejemplo, en caso de liquidación de los activos de la empresa. Todo ello será en beneficio de la empresa, de sus acreedores, de sus socios, de sus trabajadores y de la economía en su conjunto".

La Directiva también atiende a la situación de los empresarios de buena fe insolventes o sobreendeudados, que podrán gozar de la total exoneración de deudas, acogiéndose pues a una "segunda oportunidad". Tras la tormenta, siempre que su conducta haya respetado las reglas de la buena fe, podrán ver sus deudas exoneradas y podrán "recomenzar" "desde cero", "en limpio", "sobre blanco" y sin el lastre de sus deudas anteriores.

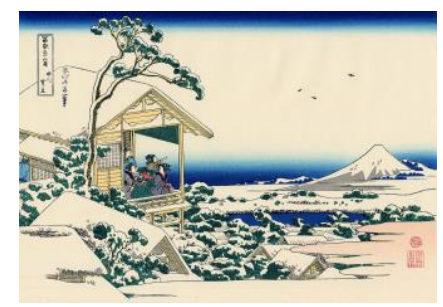

Hokusai, K., Casa de Té en Koishikawa. La mañana después de una Nevada (hacia 1830)9

\footnotetext{
${ }^{8}$ Katsushika Hokusai, Bajo el Puente Mannnen en Fukagawa (1832), File:Watermill at Onden.jpg. (2015, May 6). Wikimedia Commons, the free media repository. Retrieved 19:40, May 24, 2019 from https://commons.wikimedia.org/w/index.php?title=File:Watermill_at_Onden.jpg\&oldid=159996240.

${ }^{9}$ Hokusai, K., Casa de Té en Koishikawa. La mañana después de una Nevada (hacia 1830). File:Tea house at Koishikawa. The morning after a snowfall.jpg. (2019, February 26). Wikimedia Commons, the free media repository. Retrieved 20:09, May 24, 2019 ,

https://commons.wikimedia.org/w/index.php?title=File:Tea_house_at_Koishikawa._The_morning_after_a_snowfall.jpg\&oldid=34 $\underline{0606293 .}$.
} 
Y por fin, se diseñan en la Directiva unos procedimientos de reestructuración y de exoneración de deudas más eficaces y más ágiles que los que hay hasta ahora en los sistemas jurídicos de los Estados miembros.

\section{Conclusión}

La nueva Directiva de insolvencia de la Unión Europea de 2019 alienta la cultura del rescate de las empresas y particulares inmersos en una situación de oleaje de dificultades finanacieras, para permitir su reestructuración temprana, mientras se mantiene su actividad y evitando el concurso. Con la batería de medidas propuestas por la Unión Europea, que los Estados miembros deberán incorporar a sus Derechos internos, se aportará transparencia, seguridad jurídica y previsibilidad en la Unión Europea en beneficio de todos. Que los alumnos preuniversitarios de asignaturas económicas y jurídicas puedan conocer, aunque sea someramente, las propuestas de la normativa comunitaria y que puedan recordar, a través del recurso al arte, en este caso de Hokusai, de forma duradera, crítica y sencilla, la existencia de estas medidas legales, su contexto y su importancia, supone una innovación docente original en un campo como el jurídico en el que no se suele acudir a este medio y que puede propiciar un avance en la docencia del Derecho de las crisis de empresarios y particulares.

\section{Referencias}

Hokusai, K., La gran ola de Kanagawa (1830). The Great Wave off Kanagawa.jpg.. Wikimedia Commons, the free media repository. Retrieved 18:40, May 24, 2019 from https://commons.wikimedia.org/w/index.php?title=File:The_Great_Wave_off_Kanagawa.jpg\&ol $\underline{\mathrm{did}=350894366}$

Katsushika Hokusai, Bajo el Puente Mannnen en Fukagawa (1832), File:Watermill at Onden.jpg. Wikimedia Commons, the free media repository. Retrieved 19:40, May 24, 2019 from https://commons.wikimedia.org/w/index.php?title=File:Watermill_at_Onden.jpg\&oldid=1599962 $\underline{40}$.

Hokusai, K., Puesta de sol a través del puente Ryogoku desde la orilla del río Sumida, en Onmagayashi (1830). File:Sunset across the Ryogoku bridge from the bank of the Sumida river at Onmagayashi.jpg. (2014, July 24). Wikimedia Commons, the free media repository. https://commons.wikimedia.org/w/index.php?title=File:Sunset across the Ryogoku bridge fro m_the_bank_of_the_Sumida_river_at_Onmagayashi.jpg\&oldid=129772361.

Hokusai, K., Tormenta debajo de la cumbre (1830), File:Lightnings below the summit.jpg. Wikimedia Commons, the free media repository. Retrieved 20:24, May 24, 2019 from 
https://commons.wikimedia.org/w/index.php?title=File:Lightnings_below_the_summit.jpg\&oldid $=72583332$.

Hokusai, K., Puente colgante (1830). The Suspension Bridge on the Border of Hida and Etch $\bar{u}$ Provinces (Hietsu no sakai tsuribashi), from the series Remarkable Views of Bridges in Various Provinces (Shokoku meikyō kiran). Met. https://www.metmuseum.org/art/collection/search/53192

Hokusai, K., Casa de Té en Koishikawa. La mañana después de una Nevada (hacia 1830). File:Tea house at Koishikawa. The morning after a snowfall.jpg. Wikimedia Commons, the free media repository. $\quad$ Retrieved 20:09, May $24, \quad 2019$ from https://commons.wikimedia.org/w/index.php?title=File:Tea_house_at_Koishikawa._The_mornin g_after_a_snowfall.jpg\&oldid=340606293. 